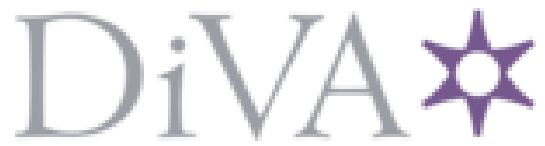

http://www.diva-portal.org

Preprint

This is the submitted version of a paper presented at IEEE International Symposium on Information Theory (ISIT), Paris, FRANCE, JUL 07-12, 2019.

Citation for the original published paper:

Vu, M T., Oechtering, T J., Skoglund, M. (2019)

Operational Equivalence of Distributed Hypothesis Testing and Identification Systems In: Proceedings 2019 IEEE International Symposium on Information Theory (ISIT) (pp. 1999-2003). IEEE https://doi.org/10.1109/ISIT.2019.8849571

N.B. When citing this work, cite the original published paper.

The work was supported by the Swedish Research Council (VR) project under Grant 2016-03853

Permanent link to this version:

http://urn.kb.se/resolve?urn=urn:nbn:se:kth:diva-263388 


\title{
Operational Equivalence of Distributed Hypothesis Testing and Identification Systems
}

\author{
Minh Thanh Vu, Tobias J. Oechtering and Mikael Skoglund \\ Division of Information Science and Engineering \\ KTH Royal Institute of Technology
}

\begin{abstract}
In this paper we revisit the connections of the distributed hypothesis testing against independence (HT) problem with the Wyner-Ahlswede-Körner (WAK) problem and the identification systems (ID). We show that the strong converse for the WAK problem is equivalent to the strong converse for the HT problem via constructive and nonconstructive transformations of codes. As another consequence of the transformation we provide a new exponentially strong converse equivalence statement. Applying the same idea, we prove a new result that the $\epsilon$-identification capacity of the ID problem is equal to the maximum $\epsilon$-exponent of type II of error in the HT problem when both side compression is allowed.
\end{abstract}

\section{INTRODUCTION}

The distributed hypothesis testing against independence (HT) and the Wyner-Ahlswede-Körner (WAK) problems are classical settings in information theory. Their achievable regions were completely characterized in [1] and [2], [3]. The strong converses, which were proved using the blowing-up lemma, were given in [1] and [4]. Recently, several new techniques have been applied to prove the strong converse [5], [6] and the exponentially strong converse [7] of the WAK problem.

Both HT and WAK problems involve sending a pair of iid sequences $\left(x^{n}, y^{n}\right)$ from different locations to a decision making center. Let $R_{c}$ be the compression rate of the sequence $x^{n} ; R_{2, \epsilon}^{\star}\left(R_{c}\right)$ be the minimum compression rate of $y^{n}$ such that $y^{n}$ can be recovered based on the compressed versions of $x^{n}$ and $y^{n}$ in the WAK problem with probability of error at most $\epsilon ; E_{\epsilon}^{\star}\left(R_{c}\right)$ be the maximum error exponent of type II of any testing scheme in the HT problem using $y^{n}$ and the compressed version of $x^{n}$ such that the probability of type I is at most $\epsilon$. The strong converse results [1], [4] state that

$$
\begin{gathered}
R_{2, \epsilon}^{\star}\left(R_{c}\right)=\min \{H(Y \mid U): U-X-Y,|\mathcal{U}| \leq|\mathcal{X}|+1, \\
\left.I(X ; U) \leq R_{c}\right\}, \forall\left(R_{c}, \epsilon\right) \in \mathbb{R}_{+} \times[0,1),
\end{gathered}
$$

and

$$
\begin{gathered}
E_{\epsilon}^{\star}\left(R_{c}\right)=\max \{I(Y ; U): U-X-Y,|\mathcal{U}| \leq|\mathcal{X}|+1, \\
\left.I(X ; U) \leq R_{c}\right\}, \forall\left(R_{c}, \epsilon\right) \in \mathbb{R}_{+} \times[0,1) .
\end{gathered}
$$

Hence, a fundamental relation between these two problems based on the strong converse results is

$$
R_{2, \epsilon}^{\star}\left(R_{c}\right)+E_{\epsilon}^{\star}\left(R_{c}\right)=H(Y), \forall\left(R_{c}, \epsilon\right) \in \mathbb{R}_{+} \times[0,1) .
$$

When $\epsilon=0$ the relation was already established via the entropy characterization in [1], [8].
Relation (3) can be interpreted as an equivalence between the strong converse for the WAK-problem and the strong converse for the HT problem. It is interesting to ask whether the relation (3) can be obtained without reproving the strong converses for both problems. We provide an affirmative answer for this question by establishing an operational relationship between these two problems. To show (3) we constructively transform any WAK-code to a corresponding HT scheme, and non-constructively show that there exists a corresponding WAK-code for any HT scheme. The corresponding probability of errors in both transformations are controllable. Interestingly, as another consequence of this transformation we also show that an exponentially strong converse of the WAK-problem is equivalent to an exponentially strong converse for the HT setting. Additionally, we present a simple example where the conditions needed to establish relation (3) holds whereas the strong converse fails since the joint iid assumption of $\left(x^{n}, y^{n}\right)$ does not hold.

The identification systems (ID) studied in [9] aim to recover the true user $w$ based on an observation sequence $y^{n}$ and a database $\left(x^{n}(i)\right)_{i=1}^{M}$, where $M$ is the number of users. $w$ is chosen uniformly at random and $y^{n}$ is related to $x^{n}(w)$. Variations of this setting were studied in [10]-[12]. Recently strong/exponentially strong converse proofs have been given for some variations in [13] and [14]. In [15] we showed a similar relation between the WAK problem and the ID problem, which we state below for convenience

$$
R_{2, \epsilon}^{\star}\left(R_{c}\right)+R_{\epsilon}^{\star}\left(R_{c}\right)=H(Y), \forall\left(R_{c}, \epsilon\right) \in \mathbb{R}_{+} \times[0,1),
$$

where $R_{\epsilon}^{\star}\left(R_{c}\right)$ is the $\epsilon$-identification capacity for a given database compression rate $R_{c}$. It is therefore natural to ask whether a direct relation between the identification problem and the hypothesis testing problem exists. Applying the same idea, we show the equivalence of the hypothesis testing against independence problem and the identification problem where compression is allowed on both sides. Namely, we constructively transform any HT scheme to an identification scheme and vice versa. We also observe that the equivalence goes beyond the assumption of iid and finite alphabets.

\section{EQUiVALENCE BETWEEN HT AND WAK PROBLEMS}

Assume that in both problems the alphabets $\mathcal{X}$ and $\mathcal{Y}$ are finite. We mainly consider the case where the source distribution is given by $X^{n} Y^{n} \sim P_{X Y}^{\otimes n}$. At the end of this section, we show an example where the main results still 
hold without the joint iid assumption. We briefly recap the definitions of a WAK-code and a HT scheme. A WAK-code consists of a pair of encoding mappings $\left(\phi_{1 n}, \phi_{2 n}\right)$ and a decoding function $\psi_{n}$ which are defined as

$$
\begin{gathered}
\phi_{1 n}: \mathcal{X}^{n} \rightarrow \mathcal{M}_{1}, \phi_{2 n}: \mathcal{Y}^{n} \rightarrow \mathcal{M}_{2} \\
\psi_{n}: \mathcal{M}_{1} \times \mathcal{M}_{2} \rightarrow \mathcal{Y}^{n} .
\end{gathered}
$$

The WAK problem aims to control $\operatorname{Pr}\left\{Y^{n} \neq \hat{Y}^{n}\right\}$, where $\hat{Y}^{n}=\psi_{n}\left(\phi_{1 n}\left(X^{n}\right), \phi_{2 n}\left(Y^{n}\right)\right)$.

The HT setting aims to differentiate between two hypotheses $H_{0}: P_{X Y}^{\otimes n}$ and $H_{1}: P_{X}^{\otimes n} \times P_{Y}^{\otimes n}$. A HT scheme consists of an encoding mapping $\phi_{n}$ and a decision mapping $\psi_{n}$, which are defined as follows

$$
\phi_{n}: \mathcal{X}^{n} \rightarrow \mathcal{M}, \psi_{n}: \mathcal{M} \times \mathcal{Y}^{n} \rightarrow\{0,1\} .
$$

The acceptance region is defined as

$$
\mathcal{A}_{n}=\left\{\left(y^{n}, m_{1}\right) \mid \psi_{n}\left(y^{n}, m_{1}\right)=0, m_{1}=\phi_{n}\left(x^{n}\right)\right\} .
$$

The probabilities of error of type I and II are given as $\alpha_{n}=$ $P_{Y^{n} \phi_{n}\left(X^{n}\right)}\left(\mathcal{A}_{n}^{c}\right)$ and $\beta_{n}=P_{Y^{n}} \times P_{\phi_{n}\left(X^{n}\right)}\left(\mathcal{A}_{n}\right)$. We present in the following an equivalent relation between a WAK-code and a HT scheme.

Theorem 1. Fix an arbitrary $\gamma>0$. Given a WAK-code $\left(\phi_{1 n}, \phi_{2 n}, \psi_{n}\right)$, we can construct a testing scheme $\left(\phi_{1 n}, \psi_{n}^{\prime}\right)$ such that the corresponding error probabilities of type I and II are given by

$$
\begin{aligned}
\alpha_{n} & \leq P_{\mathrm{WAK}}\{\text { error }\}+\operatorname{Pr}\left\{Y^{n} \notin \mathcal{A}_{\gamma}^{n}\right\} \\
\beta_{n} & \leq e^{-n(H(Y)-\gamma)}\left|\mathcal{M}_{2}\right|,
\end{aligned}
$$

where $\mathcal{A}_{\gamma}^{n}$ is the weakly typical set w.r.t $P_{Y}$. Conversely, given a testing scheme $\left(\phi_{n}, \psi_{n}\right)$ for the hypothesis testing problem there exists a WAK-code $\left(\phi_{n}, \phi_{2 n}^{\prime}, \psi_{n}^{\prime}\right)$ such that

$$
\begin{aligned}
\operatorname{Pr}\left\{\hat{Y}^{n} \neq Y^{n}\right\} & \leq \alpha_{n}+e^{n(\hat{E}-\gamma)} \beta_{n}+\operatorname{Pr}\left\{Y^{n} \notin \mathcal{A}_{\gamma}^{n}\right\} \\
& +\frac{e^{n(H(Y)+2 \gamma-\hat{E})}}{\left|\mathcal{M}_{2}\right|},
\end{aligned}
$$

where $\hat{E}$ is a free parameter that satisfies $H(Y)>\hat{E}-2 \gamma$.

Proof. WAK $\Rightarrow$ HT: For a given $m_{1} \in \mathcal{M}_{1}$, define the following correctly decodable set of the WAK-code

$$
\mathcal{D}_{m_{1}}=\left\{y^{n} \mid y^{n}=\psi_{n}\left(m_{1}, \phi_{2 n}\left(y^{n}\right)\right), y^{n} \in \mathcal{A}_{\gamma}^{n}\right\} .
$$

Then, it is clear that for all $m_{1} \in \mathcal{M}_{1}$ we have $\left|\mathcal{D}_{m_{1}}\right| \leq$ $\left|\mathcal{M}_{2}\right|$ as $\phi_{2 n}$ can only take at most $\left|\mathcal{M}_{2}\right|$ values. We use the mapping $\phi_{1 n}$ as the compression mapping for $\mathcal{X}^{n}$ in the HT problem. A decision region for the HT, based on $\mathcal{Y}^{n} \times \mathcal{M}_{1}$, is defined as

$$
\mathcal{A}_{n}=\bigcup_{m_{1}}\left(\mathcal{D}_{m_{1}} \times\left\{m_{1}\right\}\right) \subset \mathcal{Y}^{n} \times \mathcal{M}_{1} .
$$

The validity of $\mathcal{A}_{n}$, i.e., the existence of a decision mapping $\psi_{n}$ in (7), follows from the fact that we have full access to the sequence $y^{n}$ when making a decision. From (11) the probability of type I of error is bounded by

$$
\alpha_{n}=P_{Y^{n} \phi_{1 n}\left(X^{n}\right)}\left(\mathcal{A}_{n}^{c}\right) \leq P_{\mathrm{WAK}}\{\text { error }\}+\operatorname{Pr}\left\{Y^{n} \notin \mathcal{A}_{\gamma}^{n}\right\},
$$

and the probability of type II of error is bounded above by

$$
\begin{aligned}
\beta_{n} & =P_{Y^{n}} \times P_{\phi_{1 n}\left(X^{n}\right)}\left(\mathcal{A}_{n}\right)=\sum_{m_{1}} P_{\phi_{1 n}\left(X^{n}\right)}\left(m_{1}\right) P_{Y^{n}}\left(\mathcal{D}_{m_{1}}\right) \\
& \leq \sum_{m_{1}} P_{\phi_{1 n}\left(X^{n}\right)}\left(m_{1}\right)\left|\mathcal{D}_{m_{1}}\right| e^{-n(H(Y)-\gamma)} \\
& \leq e^{-n(H(Y)-\gamma)}\left|\mathcal{M}_{2}\right|
\end{aligned}
$$

WAK $\Leftarrow$ HT: Given a HT testing scheme $\left(\phi_{n}, \psi_{n}\right)$, we define the set

$\mathcal{D}_{n}\left(m_{1}\right)=\left\{y^{n} \mid \frac{1}{n} \log \frac{P_{Y^{n} \mid \phi_{n}\left(X^{n}\right)}\left(y^{n} \mid m_{1}\right)}{P_{Y^{n}}\left(y^{n}\right)}>\hat{E}-\gamma\right\} \cap \mathcal{A}_{\gamma}^{n}$.

$\mathcal{D}_{n}\left(m_{1}\right)$ plays the role of the conditional typical set in the standard proof of the WAK setting, cf. [16]. We use the mapping $\phi_{n}$ as the compression mapping for $\mathcal{X}^{n}$ in the WAKproblem. From the definition of $\mathcal{D}_{n}\left(m_{1}\right)$ we obtain

$$
\begin{aligned}
\operatorname{Pr}\left\{Y^{n}\right. & \left.\notin \mathcal{D}_{n}\left(\phi_{n}\left(X^{n}\right)\right)\right\} \\
& \stackrel{(*)}{\leq} \alpha_{n}+e^{n(\hat{E}-\gamma)} \beta_{n}+\operatorname{Pr}\left\{Y^{n} \notin \mathcal{A}_{\gamma}^{n}\right\}
\end{aligned}
$$

where $(*)$ follows from [17, Lemma 4.1.2]. Furthermore, we have $\left|\mathcal{D}_{n}\left(m_{1}\right)\right| \leq e^{n(H(Y)+2 \gamma-\hat{E})}$ for all $m_{1}$ since

$$
\begin{aligned}
1 & \geq P_{Y^{n} \mid \phi_{n}\left(X^{n}\right)}\left(\mathcal{D}_{n}\left(m_{1}\right) \mid m_{1}\right) \\
& =\sum_{y^{n} \in \mathcal{D}_{n}\left(m_{1}\right)} P_{Y^{n} \mid \phi_{n}\left(X^{n}\right)}\left(y^{n} \mid m_{1}\right) \\
& \geq \sum_{y^{n} \in \mathcal{D}_{n}\left(m_{1}\right)} P_{Y^{n}}\left(y^{n}\right) e^{n(\hat{E}-\gamma)} \\
& \geq\left|\mathcal{D}_{n}\left(m_{1}\right)\right| e^{-n(H(Y)+\gamma)} e^{n(\hat{E}-\gamma)} .
\end{aligned}
$$

Let $m_{2}$ be a uniformly random bin index of $y^{n}$ and $\mathcal{B}\left(m_{2}\right)$ be the set of all such $y^{n}$. The decoder decides that $\hat{y}^{n}$ is the reconstructed sequence if it is the unique sequence such that $\hat{y}^{n} \in \mathcal{B}\left(m_{2}\right) \cap \mathcal{D}_{n}\left(m_{1}\right)$, where $m_{1}$ and $m_{2}$ are sent messages from Encoder 1 and 2. It then follows that

$$
\begin{aligned}
& \operatorname{Pr}\left\{\hat{Y}^{n} \neq Y^{n}\right\} \leq \operatorname{Pr}\left\{Y^{n} \notin \mathcal{B}\left(M_{2}\right) \cap \mathcal{D}_{n}\left(\phi_{n}\left(X^{n}\right)\right)\right\} \\
& +\operatorname{Pr}\left\{\exists \tilde{y}^{n} \neq Y^{n}, \tilde{y}^{n} \in \mathcal{D}_{n}\left(\phi_{n}\left(X^{n}\right)\right) \cap \mathcal{B}\left(M_{2}\right)\right\} \\
& \stackrel{(a)}{\leq} \operatorname{Pr}\left\{Y^{n} \notin \mathcal{D}_{n}\left(\phi_{n}\left(X^{n}\right)\right)\right\} \\
& +\operatorname{Pr}\left\{\exists \tilde{y}^{n} \neq Y^{n}, \tilde{y}^{n} \in \mathcal{D}_{n}\left(\phi_{n}\left(X^{n}\right)\right) \cap \mathcal{B}\left(M_{2}\right)\right\} \\
& \stackrel{(b)}{\leq} \alpha_{n}+e^{n(\hat{E}-\gamma)} \beta_{n}+\operatorname{Pr}\left\{Y^{n} \notin \mathcal{A}_{\gamma}^{n}\right\}+\frac{e^{n(H(Y)+2 \gamma-\hat{E})}}{\left|\mathcal{M}_{2}\right|} .
\end{aligned}
$$

(a) is valid since $Y^{n} \in \mathcal{B}\left(M_{2}\right)$. The inequality (b) holds since each $\tilde{y}^{n}$ is assigned independently to a bin with probability $1 /\left|\mathcal{M}_{2}\right|$ and the number of such $\tilde{y}^{n}$ is bounded by $e^{n(H(Y)+2 \gamma-\hat{E})}$, cf. (14). The existence of deterministic mappings $\phi_{2 n}^{\prime}$ and $\psi_{n}^{\prime}$ follows immediately. 
Remark 1. Note that if a randomized test is used for the HT, then the second part of Theorem 1 is still valid since $(*)$ in (13) holds due to [18. Lemma 12.2].

Before proceeding further we need definitions of $\epsilon$ achievable regions. Fix an $\epsilon \in[0,1)$.

Definition 1. Let $\mathcal{R}_{\mathrm{WAK}, \epsilon}$ be the closure of all $\left(R_{1}, R_{2}\right)$ such that there exists a WAK-code $\left(\phi_{1 n}, \phi_{2 n}, \psi_{n}\right)$ which satisfies

$$
\begin{aligned}
& \limsup _{n \rightarrow \infty} \frac{1}{n} \log \left|\mathcal{M}_{i}\right| \leq R_{i}, i=1,2, \\
& \limsup _{n \rightarrow \infty} \operatorname{Pr}\left\{\hat{Y}^{n} \neq Y^{n}\right\} \leq \epsilon .
\end{aligned}
$$

Define $R_{2, \epsilon}^{\star}\left(R_{1}\right)=\inf \left\{R_{2} \mid\left(R_{1}, R_{2}\right) \in \mathcal{R}_{\mathrm{WAK}, \epsilon}\right\}$.

As $\left(R_{1}, H(Y)\right) \in \mathcal{R}_{\mathrm{WAK}, \epsilon}, \forall \epsilon \in[0,1), R_{2, \epsilon}^{\star}\left(R_{1}\right)$ is finite. Additionally, since $\mathcal{R}_{\mathrm{WAK}, \epsilon}$ is a closed set, the $\inf \{\cdot\}$ operation in the definition of $R_{2, \epsilon}^{\star}\left(R_{1}\right)$ can be replaced by the $\min \{\cdot\}$ operation. Additionally, let us define the following:

Definition 2. Let $\mathcal{R}_{\mathrm{HT}, \epsilon}$ be the closure of all $\left(R_{c}, E\right)$ such that there exists a testing scheme $\left(\phi_{n}, \psi_{n}\right)$ which satisfies

$$
\begin{gathered}
\limsup _{n \rightarrow \infty} \frac{1}{n} \log \left|\mathcal{M}_{1}\right| \leq R_{c}, \limsup _{n \rightarrow \infty} \alpha_{n} \leq \epsilon, \\
\liminf _{n \rightarrow \infty} \frac{1}{n} \log \frac{1}{\beta_{n}} \geq E .
\end{gathered}
$$

Define $E_{\epsilon}^{\star}\left(R_{c}\right)=\sup \left\{E \mid\left(R_{c}, E\right) \in \mathcal{R}_{\mathrm{HT}, \epsilon}\right\}$.

We note that $E_{\epsilon}^{\star}\left(R_{c}\right) \leq I(X ; Y)$ since the latter is the error exponent of uncompressed data [17, Corollary 4.2.1]. Additionally, $E_{\epsilon}^{\star}\left(R_{c}\right) \geq 0$ also holds since $\left(R_{c}, 0\right) \in \mathcal{R}_{\mathrm{HT}, \epsilon}$, $\forall \epsilon \in[0,1)$.

As a consequence of Theorem 1 we prove $(3)$ in the following result.

Theorem 2. For all $R_{c} \in \mathbb{R}_{+}$and for all $\epsilon \in[0,1)$ we have

$$
E_{\epsilon}^{\star}\left(R_{c}\right)+R_{2, \epsilon}^{\star}\left(R_{c}\right)=H(Y) .
$$

Proof. Given a $\gamma>0$ and an $\epsilon>0$, there exists a WAK-code $\left(\phi_{1 n}, \phi_{2 n}, \psi_{n}\right)$ which satisfies all the conditions in Definition 1 for the rate pair $\left(R_{c}+\gamma, R_{2, \epsilon}^{\star}\left(R_{c}\right)+\gamma\right)$. This implies that for all sufficiently large $n$ we have $\left|\mathcal{M}_{2}\right| \leq e^{n\left(R_{2, \epsilon}^{\star}\left(R_{c}\right)+2 \gamma\right)}$. By the first part of Theorem 11, the corresponding testing scheme satisfies

$\limsup _{n \rightarrow \infty} \alpha_{n} \leq \epsilon, \liminf _{n \rightarrow \infty} \frac{1}{n} \log \frac{1}{\beta_{n}} \geq H(Y)-3 \gamma-R_{2, \epsilon}^{\star}\left(R_{c}\right)$.

This implies that $\left(R_{c}+\gamma, H(Y)-3 \gamma-R_{2, \epsilon}^{\star}\left(R_{c}\right)\right) \in \mathcal{R}_{\mathrm{HT}, \epsilon}$. Since $\gamma>0$ is arbitrary and $\mathcal{R}_{\mathrm{HT}, \epsilon}$ is closed by definition, we obtain $E_{\epsilon}^{\star}\left(R_{c}\right) \geq H(Y)-R_{2, \epsilon}^{\star}\left(R_{c}\right)$.

Conversely, given a $\gamma>0$ and an $\epsilon>0$, there exists a testing scheme $\left(\phi_{n}, \psi_{n}\right)$ such that $\left(R_{c}+\gamma, E_{\epsilon}^{\star}\left(R_{c}\right)-\gamma\right)$ is $\epsilon$-achievable according to Definition 2 i.e., for all sufficiently large $n$ we have $\beta_{n} \leq e^{-n\left(E_{\epsilon}^{\star}\left(R_{c}\right)-2 \gamma\right)}$. Choosing $\hat{E}=E_{\epsilon}^{\star}\left(R_{c}\right)-2 \gamma$ and $\left|\mathcal{M}_{2}\right|=e^{n\left(H(Y)+5 \gamma-E_{\epsilon}^{\star}\left(R_{c}\right)\right)}$ we obtain by the second part of Theorem 1 that

$$
\limsup _{n \rightarrow \infty} \operatorname{Pr}\left\{\hat{Y}^{n} \neq Y^{n}\right\} \leq \epsilon,
$$

which implies that $\left(R_{c}+\gamma, H(Y)+5 \gamma-E_{\epsilon}^{\star}\left(R_{c}\right)\right) \in \mathcal{R}_{\mathrm{WAK}, \epsilon}$. Since $\gamma$ is arbitrary and $\mathcal{R}_{\mathrm{WAK}, \epsilon}$ is also closed by definition, we therefore obtain $R_{2, \epsilon}^{\star}\left(R_{c}\right) \leq H(Y)-E_{\epsilon}^{\star}\left(R_{c}\right)$.

Another important consequence of Theorem 1 , which states an equivalence of exponentially strong converse statements, is given as follows.

Theorem 3. The following statements are equivalent:

1) For any code which satisfies $\lim \sup _{n \rightarrow \infty} \frac{1}{n} \log \left|\mathcal{M}_{1}\right| \leq R_{c}$ and $\limsup _{n \rightarrow \infty} \frac{1}{n} \log \left|\mathcal{M}_{2}\right| \leq R_{2}$ in the WAK problem, if $\left(R_{c}, R_{2}\right) \notin \mathcal{R}_{\mathrm{WAK}, 0}$, then $\operatorname{Pr}\left\{Y^{n} \neq \hat{Y}^{n}\right\} \rightarrow 1$ exponentially fast at a positive convergence rate.

2) For any $H T$ scheme with $\lim \sup _{n \rightarrow \infty} \frac{1}{n} \log \left|\mathcal{M}_{1}\right| \leq R_{c}$ and $\liminf \operatorname{in}_{n \rightarrow \infty} \frac{1}{n} \log \frac{1}{\beta_{n}} \geq E$, if $\left(R_{c}, E\right) \notin \mathcal{R}_{\mathrm{HT}, 0}$, then $\alpha_{n} \rightarrow 1$ exponentially fast at a positive convergence rate.

A proof of the first statement is presented in [7].

Proof. Assume that the first statement holds. It suffices to show the second statement when $E<H(Y)$. Let $\left(\phi_{n}, \psi_{n}\right)$ be a hypothesis testing scheme such that $\lim \sup _{n \rightarrow \infty} \frac{1}{n} \log M \leq$ $R_{c}$ and $\liminf _{n \rightarrow \infty} \frac{1}{n} \log \frac{1}{\beta_{n}} \geq E$ where $\left(R_{c}, E\right) \notin \mathcal{R}_{\mathrm{HT}, 0}$. Let $\gamma>0$ be small enough such that $\left(R_{c}, H(Y)+4 \gamma-E\right) \notin$ $\mathcal{R}_{\mathrm{WAK}, 0}$. By the second part of Theorem 1, there exists a WAK-code $\left(\phi_{n}, \phi_{2 n}^{\prime}, \psi_{n}^{\prime}\right)$ such that with $E=E-\gamma$ and $\left|\mathcal{M}_{2}\right|=e^{n(H(Y)+4 \gamma-E)}$ we have

$$
\operatorname{Pr}\left\{\hat{Y}^{n} \neq Y^{n}\right\} \leq \alpha_{n}+2 e^{-n \gamma}+\operatorname{Pr}\left\{Y^{n} \notin \mathcal{A}_{\gamma}^{n}\right\} .
$$

Since the weakly typical set $\mathcal{A}_{\gamma}^{n}$ includes the strongly typical set $\mathcal{T}_{\epsilon}^{n}$ for a fixed, positive, and small enough $\epsilon$, the last term goes to 0 exponentially with a convergence rate of at least $2 \epsilon^{2}$. By the assumption $\operatorname{Pr}\left\{Y^{n}=\hat{Y}^{n}\right\}$ goes to 0 exponentially at a rate of $\eta>0$, we then have $\alpha_{n} \rightarrow 1$ exponentially at a rate of $\min \left\{\eta, \gamma, 2 \epsilon^{2}\right\}$.

Conversely, assume that the second statement holds. Let $\left(\phi_{1 n}, \phi_{2 n}, \psi_{n}\right)$ be a WAK-code such that

$$
\limsup _{n \rightarrow \infty} \frac{1}{n} \log \left|\mathcal{M}_{1}\right| \leq R_{c}, \limsup _{n \rightarrow \infty} \frac{1}{n} \log \left|\mathcal{M}_{2}\right| \leq R_{2},
$$

where $\left(R_{c}, R_{2}\right) \notin \mathcal{R}_{\mathrm{WAK}, 0}$. Let $\gamma>0$ be small enough such that $\left(R_{c}, H(Y)-2 \gamma-R_{2}\right) \notin \mathcal{R}_{\mathrm{HT}, 0}$. By the first part of Theorem 11. the constructed testing scheme satisfies $\lim \inf \frac{1}{n} \log \frac{1}{\beta_{n}} \geq H(Y)-2 \gamma-R_{2}$. The corresponding false alarm probability $\alpha_{n}$ hence goes to 1 exponentially at a rate of $\xi>0$, or $P_{\mathrm{WAK}}\{$ error $\} \rightarrow 1$ exponentially at a rate of $\min \left\{\xi, 2 \epsilon^{2}\right\}$.

Remark 2. Note that the following two conditions are needed for proof of Theorems 1 and 2. $P_{Y^{n}}=P_{Y}^{\otimes n}$ to ensure that $2^{-n(H(Y)+\gamma)} \leq P_{Y^{n}}\left(y^{n}\right) \leq 2^{-n(H(Y)-\gamma)},\left(R_{1}, H(Y)\right) \in$ $\mathcal{R}_{\mathrm{WAK}, \epsilon}$ as well as $\operatorname{Pr}\left\{Y^{n} \notin \mathcal{A}_{\gamma}^{n}\right\} \rightarrow 0$, and $E^{\star}\left(R_{c}\right)$ is upper bounded by $H(Y)$. We present now a simple example on finite alphabets without the iid assumption of the joint distribution where these two conditions are still active. Hence, Theorem 2 is still valid. Let $P_{1, X Y}$ and $P_{2, X Y}$ be distributions which belong to the set $\left\{Q_{X Y} \mid Q_{X}=P_{X}, Q_{Y}=P_{Y}\right\}$ such that $D\left(P_{1, X Y} \| P_{X} \times P_{Y}\right)<D\left(P_{2, X Y} \| P_{X} \times P_{Y}\right)$. Let the source 
distribution of the WAK problem and the distribution under $H_{0}$ in the HT problem be $P_{X^{n} Y^{n}}=\alpha P_{1, X Y}^{\otimes n}+(1-\alpha) P_{2, X Y}^{\otimes n}$ where $\alpha \in(0,1)$. The distribution under $H_{1}$ is still $P_{X}^{\otimes n} \times P_{Y}^{\otimes n}$. The process governed by $P_{X^{n} Y^{n}}$ is stationary but nonergodic, cf. [17. Fig. 1.5] for an illustration of its information-spectrum. The maximum error exponent of type II in the uncompressed case is given by [17 Example 4.2.1]

$$
E_{\epsilon}^{\star}(\log |\mathcal{X}|)=\left\{\begin{array}{ll}
D\left(P_{1, X Y} \| P_{X} \times P_{Y}\right) & \text { if } 0 \leq \epsilon<\alpha \\
D\left(P_{2, X Y} \| P_{X} \times P_{Y}\right) & \text { when } \alpha \leq \epsilon<1
\end{array} .\right.
$$

Note that $E_{\epsilon}^{\star}(\log |\mathcal{X}|) \leq H(Y)$ holds. The strong converse however does not hold.

\section{EQUIVALENCE BETWEEN HT AND IDENTIFICATION}

We establish herein the equivalence between the hypothesis testing against independence and the identification problem. Two hypotheses in the HT problem are $H_{0}: P_{X^{n} Y^{n}}$, $H_{1}: P_{X^{n}} \times P_{Y^{n}}$. Note that we do not assume either $P_{X^{n} Y^{n}}=$ $P_{X Y}^{\otimes n}$, or $\mathcal{X}$ and $\mathcal{Y}$ are finite. However, it should be assumed that $\mathcal{X}$ and $\mathcal{Y}$ are nice enough, for example Polish spaces. Since compression of $y^{n}$ is allowed in this section, we redefine some terms and notation in the following.

A testing scheme consists of two compression mappings $\left(\phi_{1 n}, \phi_{2 n}\right)$ and a decision mapping $\psi_{n}$ where $\phi_{1 n}: \mathcal{X}^{n} \rightarrow$ $\mathcal{M}_{1}, \phi_{2 n}: \mathcal{Y}^{n} \rightarrow \mathcal{M}_{2}$ and $\psi_{n}: \mathcal{M}_{1} \times \mathcal{M}_{2} \rightarrow\{0,1\}$. The acceptance region can be defined similarly as in (7). The probabilities of type I and II errors $\alpha_{n}$ and $\beta_{n}$ can also be determined accordingly. This setup was discussed briefly in [1], for which a single-letter characterization for the optimal achievable error exponent of type II of error is still challenging.

For a detailed introduction of the identification systems the reader is referred to the works [9], [11]. Given $M$ users in the database, we assume that each data sequence $x^{n}(i)$, where $i \in[1: M]$, is generated independently from a common distribution $P_{X^{n}}$. An observation sequence $y^{n}$ is observed at the output of a channel $P_{Y^{n} \mid X^{n}}$ with the input $x^{n}(w)$ where $w$ is chosen uniformly at random from $[1: M]$. Hence, the joint distribution of the users' information, the observation and the randomly selected index in the ID problem is given as

$$
\begin{aligned}
& P_{Y^{n}\left\{X^{n}(i)\right\}_{i=1}^{M} W}\left(y^{n},\left\{x^{n}(i)\right\}_{i=1}^{M}, w\right) \\
& =\frac{1}{M} P_{Y^{n} \mid X^{n}}\left(y^{n} \mid x^{n}(w)\right) \times \prod_{k} P_{X^{n}}\left(x^{n}(k)\right) .
\end{aligned}
$$

An identification scheme consists of two compression mappings $\left(\phi_{1 n}, \phi_{2 n}\right)$ and a decoding mapping $\psi_{n}$ where

$$
\begin{aligned}
\phi_{1 n}: \mathcal{X}^{n} & \rightarrow \mathcal{M}_{1}, \phi_{2 n}: \mathcal{Y}^{n} \rightarrow \mathcal{M}_{2} \\
\psi_{n} & : \mathcal{M}_{1}^{M} \times \mathcal{M}_{2} \rightarrow \mathcal{W} \cup\{e\} .
\end{aligned}
$$

In the identification problem one wants to control the probability of incorrect identification $\operatorname{Pr}\{\hat{W} \neq W\}$, where $\hat{W}=$ $\psi_{n}\left(\left\{\phi_{1 n}\left(X^{n}(i)\right)\right\}_{i=1}^{M}, \phi_{2 n}\left(Y^{n}\right)\right)$. This setting was studied in [12], where inner bounds and outer bounds on the achievable rate region were derived. A connection between the achievable regions of these two problems has been drawn recently in [19] via the entropy characterization.

We first establish the following useful lemma, which is a generalization of [15, Lemma 3].

Lemma 1. For a given $\gamma>0$ and a given identification scheme $\left(\phi_{1 n}, \phi_{2 n}, \psi_{n}\right)$, we have

$$
\begin{aligned}
& \operatorname{Pr}\{\hat{W} \neq W\} \geq \operatorname{Pr}\left\{\iota\left(\phi_{2 n}\left(\bar{Y}^{n}\right) ; \phi_{1 n}\left(\bar{X}^{n}\right)\right) \leq \log M-n \gamma\right\} \\
& \quad-e^{-n \gamma}, \\
& \text { where }\left(\bar{Y}^{n}, \bar{X}^{n}\right) \sim P_{Y^{n} X^{n}} \text { and } \\
& \qquad \iota\left(\phi_{2 n}\left(y^{n}\right) ; \phi_{1 n}\left(x^{n}\right)\right) \\
& \qquad=\log \frac{P_{\phi_{2 n}\left(\bar{Y}^{n}\right) \mid \phi_{1 n}\left(\bar{X}^{n}\right)}}{P_{\phi_{2 n}\left(\bar{Y}^{n}\right)}}\left(\phi_{2 n}\left(y^{n}\right), \phi_{1 n}\left(x^{n}\right)\right) .
\end{aligned}
$$

It can be seen that the first term on the right-hand side of Lemma 1 is the corresponding false alarm probability when testing $H_{0}: P_{X^{n} Y^{n}}$ versus $H_{1}: P_{X^{n}} \times P_{Y^{n}}$ using the loglikelihood ratio test with the corresponding threshold $\log M-$ $n \gamma$. The proof of Lemma 1 can be found in [20, Appendix B]. Using Lemma 1 we can establish an analogue of Theorem 1 as follows.

Theorem 4. Fix an arbitrary $\gamma>0$. Given an identification scheme $\left(\phi_{1 n}, \phi_{2 n}, \psi_{n}\right)$, we can construct a HT scheme $\left(\phi_{1 n}, \phi_{2 n}, \psi_{n}^{\prime}\right)$ such that the corresponding error probabilities of type I and II are given by

$$
\alpha_{n} \leq \operatorname{Pr}\{\hat{W} \neq W\}+e^{-n \gamma}, \quad \beta_{n} \leq \frac{e^{n \gamma}}{M} .
$$

Conversely, given a testing scheme $\left(\phi_{1 n}, \phi_{2 n}, \psi_{n}\right)$ for the HT problem, we can construct an identification scheme $\left(\phi_{1 n}, \phi_{2 n}, \psi_{n}^{\prime}\right)$ such that

$$
\operatorname{Pr}\{\hat{W} \neq W\} \leq \alpha_{n}+M \beta_{n} .
$$

Proof. ID $\Rightarrow$ HT: From a given identification scheme $\left(\phi_{1 n}, \phi_{2 n}, \psi_{n}\right)$ we use the same pair of mappings $\left(\phi_{1 n}, \phi_{2 n}\right)$ to compress information in the HT. From the interpretation of the Lemma 2 given above, it is natural to define an acceptance region for the HT setup as

$$
\begin{aligned}
\mathcal{A}_{n}=\{ & \left(\phi_{1 n}\left(x^{n}\right), \phi_{2 n}\left(y^{n}\right)\right) \mid \\
& \left.\iota\left(\phi_{2 n}\left(y^{n}\right) ; \phi_{1 n}\left(x^{n}\right)\right)>\log M-n \gamma\right\} .
\end{aligned}
$$

The probability of type I of error is then given by

$$
\alpha_{n}=P_{\phi_{1 n}\left(\bar{X}^{n}\right) \phi_{2 n}\left(\bar{Y}^{n}\right)}\left(\mathcal{A}_{n}^{c}\right) \leq \operatorname{Pr}\{\hat{W} \neq W\}+e^{-n \gamma},
$$

where the inequality follows from Lemma 1 By the change of measure we also obtain

$$
\beta_{n}=P_{\phi_{1 n}\left(\bar{X}^{n}\right)} \times P_{\phi_{2 n}\left(\bar{Y}^{n}\right)}\left(\mathcal{A}_{n}\right) \leq \frac{e^{n \gamma}}{M} .
$$

ID $\Leftarrow$ HT: Given a testing scheme $\left(\phi_{1 n}, \phi_{2 n}, \psi_{n}\right)$ of the HT, let $\mathcal{A}_{n}$ be the acceptance region. We use the mapping $\phi_{1 n}$ to compress each user's information and store it into a database and the mapping $\phi_{2 n}$ to compress the observation sequence $y^{n}$ in the identification setting. We define the decoding rule as follows. We search for a unique $\hat{w}$ such that 
$\left(\phi_{2 n}\left(y^{n}\right), \phi_{1 n}\left(x^{n}(\hat{w})\right)\right) \in \mathcal{A}_{n}$. If there exists none or there is more than one of such index, we output $e$. The analysis is standard hence omitted. The interested reader is referred to [20, p.13].

We need some additional definitions. For an arbitrary but fixed $\epsilon \in[0,1)$, define $\mathcal{R}_{\mathrm{ID}, \epsilon}$ to be the closure of all tuples $\left(R_{1}, R_{2}, R\right)$ such that there exists an identification scheme $\left(\phi_{1 n}, \phi_{2 n}, \psi_{n}\right)$ which satisfies

$$
\begin{aligned}
\limsup _{n \rightarrow \infty} \frac{1}{n} \log \left|\mathcal{M}_{i}\right| \leq R_{i}, i=1,2, \\
\quad \liminf _{n \rightarrow \infty} \frac{1}{n} \log M \geq R, \limsup _{n \rightarrow \infty} \operatorname{Pr}\{\hat{W} \neq W\} \leq \epsilon .
\end{aligned}
$$

Then we can define the $\epsilon$-identification capacity for a given compression rate pair $\left(R_{1}, R_{2}\right)$ as $R_{\epsilon}^{\star}\left(R_{1}, R_{2}\right)=\sup \{R \mid$ $\left.\left(R_{1}, R_{2}, R\right) \in \mathcal{R}_{\mathrm{ID}, \epsilon}\right\}$. Further, define $\mathcal{R}_{\mathrm{HT}, \epsilon}$ to be the closure of all tuples $\left(R_{1}, R_{2}, E\right)$ such that there exists a HT scheme $\left(\phi_{1 n}, \phi_{2 n}, \psi_{n}\right)$ such that

$$
\begin{array}{r}
\limsup _{n \rightarrow \infty} \frac{1}{n} \log \left|\mathcal{M}_{i}\right| \leq R_{i}, i=1,2, \\
\limsup _{n \rightarrow \infty} \alpha_{n} \leq \epsilon, \liminf _{n \rightarrow \infty} \frac{1}{n} \log \frac{1}{\beta_{n}} \geq E .
\end{array}
$$

Similarly we define the maximum $\epsilon$ - achievable error exponent for a given compression rate pair $\left(R_{1}, R_{2}\right)$ as $E_{\epsilon}^{\star}\left(R_{1}, R_{2}\right)=$ $\sup \left\{E \mid\left(R_{1}, R_{2}, E\right) \in \mathcal{R}_{\mathrm{HT}, \epsilon}\right\}$. A connection between $\epsilon$ achievable rate regions of the two settings is given as follows.

Theorem 5. For all $\epsilon \in[0,1)$ and for all $\left(R_{1}, R_{2}\right) \in \mathbb{R}_{+}^{2}$, the following equality holds $E_{\epsilon}^{\star}\left(R_{1}, R_{2}\right)=R_{\epsilon}^{\star}\left(R_{1}, R_{2}\right)$.

Proof. Assume that both quantities are finite. Given a $\gamma>$ 0 , there exists an identification scheme $\left(\phi_{1 n}, \phi_{2 n}, \psi_{n}\right)$ such that all conditions in 29) are satisfied for $\left(R_{1}+\gamma, R_{2}+\right.$ $\left.\gamma, R_{\epsilon}^{\star}\left(R_{1}, R_{2}\right)-\gamma\right)$. This implies that for all sufficiently large $n$ we have $M \geq e^{n\left(R_{\epsilon}^{\star}\left(R_{1}, R_{2}\right)-2 \gamma\right)}$. Then by the first part of Theorem 4 the probabilities of error of the corresponding HT scheme are bounded by

$$
\limsup _{n \rightarrow \infty} \alpha_{n} \leq \epsilon, \liminf _{n \rightarrow \infty} \frac{1}{n} \log \frac{1}{\beta_{n}} \geq R_{\epsilon}^{\star}\left(R_{1}, R_{2}\right)-3 \gamma .
$$

This implies that $E_{\epsilon}^{\star}\left(R_{1}, R_{2}\right) \geq R_{\epsilon}^{\star}\left(R_{1}, R_{2}\right)$, by taking $\gamma \rightarrow 0$.

Conversely, there exists a testing scheme $\left(\phi_{1 n}, \phi_{2 n}, \psi_{n}\right)$ such that all conditions in (30) are satisfied for $\left(R_{1}+\gamma, R_{2}+\right.$ $\left.\gamma, E_{\epsilon}^{\star}\left(R_{1}, R_{2}\right)-\gamma\right)$. This implies that for all sufficiently large $n$ we have $\beta_{n} \leq e^{-n\left(E_{\epsilon}^{*}\left(R_{1}, R_{2}\right)-2 \gamma\right)}$. By choosing $M=$ $e^{n\left(E_{\epsilon}^{\star}\left(R_{1}, R_{2}\right)-3 \gamma\right)}$ we obtain from the second part of Theorem 4

$$
\limsup _{n \rightarrow \infty} \operatorname{Pr}\{\hat{W} \neq W\} \leq \epsilon,
$$

which implies that $\left(R_{1}+\gamma, R_{2}+\gamma, E_{\epsilon}^{\star}\left(R_{1}, R_{2}\right)-3 \gamma\right) \in \mathcal{R}_{\mathrm{ID}, \epsilon}$. Hence, we have $E_{\epsilon}^{\star}\left(R_{1}, R_{2}\right) \leq R_{\epsilon}^{\star}\left(R_{1}, R_{2}\right)$.

Next, if $E_{\epsilon}^{\star}\left(R_{1}, R_{2}\right)=\infty$ for some pair $\left(R_{1}, R_{2}\right) \in \mathbb{R}_{+}^{2}$ and $\epsilon>0$, then we can modify the proof as follows: Let $\left\{E_{m}\right\}_{m=1}^{\infty}$ be a sequence such that $E_{m}<\infty, \forall m$, and $E_{m} \rightarrow \infty$ as $m \rightarrow \infty$. Then we replace $E_{\epsilon}^{\star}\left(R_{1}, R_{2}\right)$ with $E_{m}$ in the last paragraph to get $\left(R_{1}, R_{2}, E_{m}\right) \in \mathcal{R}_{\mathrm{ID}, \epsilon}$. This holds for any $m$, hence $R_{\epsilon}^{\star}\left(R_{1}, R_{2}\right)=\infty$ as well. The case $R_{\epsilon}^{\star}\left(R_{1}, R_{2}\right)=\infty$ can be handled similarly.

Remark 3. In [15. Appendix E] we showed a strong converse proof of the iid Gaussian identification system without compression of $y^{n}$. As the consequence of Theorem 5 it implies a strong converse proof of the HT against independence for the iid Gaussian scenario. Note further that we can replace $n \gamma$ in Lemma 1 with $\sqrt{n} \gamma$. Hence, we could establish a secondorder relation between these two settings as follows. If in the first part of Theorem 4 we have $M \geq e^{n(R+S / \sqrt{n})}$ then we obtain $\beta_{n} \leq e^{-n(R+(S-\gamma) / \sqrt{n})}$. If in the second part of Theorem 4 we have $\beta_{n} \leq e^{-n(E+S / \sqrt{n})}$ then we can choose $M=e^{n(E+(S-\gamma) / \sqrt{n})}$.

\section{REFERENCES}

[1] R. Ahlswede and I. Csiszár, "Hypothesis testing with communication constraints," IEEE Trans. Inf. Theory, vol. 32, no. 4, pp. 533-542, 1986.

[2] A. Wyner, "On source coding with side information at the decoder," IEEE Trans. Inf. Theory, vol. 21, no. 3, pp. 294-300, 1975.

[3] R. Ahlswede and J. Körner, "Source coding with side information and a converse for degraded broadcast channels," IEEE Trans. Inf. Theory, vol. 21, no. 6, pp. 629-637, 1975.

[4] R. Ahlswede, P. Gács, and J. Körner, "Bounds on conditional probabilities with applications in multi-user communication," Zeitschrift für Wahrscheinlichkeitstheorie und verwandte Gebiete, vol. 34, no. 2, pp. 157-177, 1976.

[5] S. Watanabe, "A converse bound on Wyner-Ahlswede-Körner network via Gray-Wyner network," in Information Theory Workshop (ITW), 2017 IEEE, 2017, pp. 81-85.

[6] J. Liu, R. van Handel, and S. Verdú, "Beyond the blowing-up lemma: Sharp converses via reverse hypercontractivity," in 2017 IEEE International Symposium on Information Theory (ISIT), 2017, pp. 943-947.

[7] Y. Oohama, "Exponent function for one helper source coding problem at rates outside the rate region," in 2015 IEEE International Symposium on Information Theory (ISIT), 2015, pp. 1575-1579.

[8] C. Tian and J. Chen, "Successive refinement for hypothesis testing and lossless one-helper problem," IEEE Trans. Inf. Theory, vol. 54, no. 10, pp. 4666-4681, 2008.

[9] F. M. J. Willems, T. Kalker, and J.-P. Linnartz, "On the capacity of a biometrical identification system," in International Symposium on Information Theory, 2003.

[10] E. Tuncel, "Capacity/storage tradeoff in high-dimensional identification systems," IEEE Trans. Inf. Theory, vol. 55, no. 5, pp. 2097-2106, 2009.

[11] E. Tuncel and D. Gündüz, "Identification and lossy reconstruction in noisy databases," IEEE Trans. Inf. Theory, vol. 60, no. 2, pp. 822-831, 2014.

[12] M. B. Westover and J. A. O'Sullivan, "Achievable rates for pattern recognition," IEEE Trans. Inf. Theory, vol. 54, no. 1, pp. 299-320, 2008.

[13] L. Zhou, V. Y. Tan, L. Yu, and M. Motani, "Exponential strong converse for content identification with lossy recovery," IEEE Trans. Inf. Theory, 2018.

[14] V. Yachongka and H. Yagi, "Reliability function and strong converse of biomedical identification systems," in International Symposium on Information Theory and Its Applications (ISITA). IEEE, 2016, pp. $547-551$.

[15] M. T. Vu, T. J. Oechtering, M. Skoglund, and H. Boche, "Uncertainty in identification systems," Submitted.

[16] T. M. Cover and J. A. Thomas, Elements of information theory. John Wiley \& Sons, 2012.

[17] T. S. Han, Information-Spectrum Methods in Information Theory. Springer-Verlag Berlin Heidelberg, 2003.

[18] Y. Polyanskiy and Y. Wu, "Lecture notes on information theory," MIT (6.441), UIUC (ECE 563), 2017.

[19] G. Pichler, P. Piantanida, and G. Matz, "Distributed informationtheoretic clustering," arXiv preprint arXiv:1602.04605, 2016.

[20] M. T. Vu, T. J. Oechtering, and M. Skoglund, "Hypothesis testing and identification systems," Submitted. 